\title{
Variation of energy absorption build-up factor with penetration depth for some titanium compounds
}

\author{
Lovedeep singh $^{1, \text { a }}$, Pooja Rani ${ }^{1}$ and SS Ghumman ${ }^{1}$ \\ ${ }^{1}$ Sant Longowal Institute of Engineering and Technology, Longowal \\ Punjab, India
}

\begin{abstract}
The buildup factor is an important characteristic that need to be studied and determined prior to using a material clinically in radiation treatment and protection. Energy absorption buildup factors for some titanium compounds like Titanium dioxide $\left(\mathrm{TiO}_{2}\right)$, Titanium Carbide (TiC), Titanium Nitride (TiN) and Titanium Silicate $\left(\mathrm{TiSi}_{2}\right)$ has been calculated using the G.P. fitting technique upto penetration depth of 40 mean free path (mfp) and in an energy range from 0.015 to $15.0 \mathrm{MeV}$. The variation of absorption buildup factors with penetration depth for the selected compounds of titanium has been studied. It has been found that the maximum value of energy absorption buildup factors shifts to the intermediate incident photon energy regions with the increase in penetration depth of the selected compounds of titanium.
\end{abstract}

\section{Keywords:}

Titanium compounds, multiple scattering, buildup factor.

\section{INTRODUCTION}

Gamma rays are electromagnetic radiations having wavelength range of about $10^{-14}$ to $10^{-1} \mathrm{~m}$. These are the most energetic electromagnetic radiations. Due to their greater penetrating power, interactions of gamma ray photons with matter achieved prime importance and interest. Also titanium is the $9^{\text {th }}$ most abundant element in the nature having properties of high hardness, brightness and strongness. It is widely used in aerospace, sports and medicine fields. Titanium compounds are also found useful in various fields. They have applications in paint, coloring food and cosmetics, crayons, UV protection fields, lubricants, wear resistant tools and many more. Due to so many applications of compounds of titanium, an attempt has been made to study their interaction with gamma rays and hence to check their possibility in radiation shielding. Radiation physicists face a main problem of leakage of radiation due to the Compton multiple scattering events. This multiple scattering is the main reason for violation of the Lambert-Beer law i.e. $\mathrm{I}=$ $\mathrm{I}_{0} \mathrm{e}^{\mu \mathrm{x}}$. In order to maintain this law, a correction factor B is used. This B is called the buildup factor which measures the degree up to which the Lambert-Beer law is violated. Then the intensity equation after modification becomes $\mathrm{I}=\mathrm{BI}_{\mathrm{o}} \mathrm{e}^{\mu \mathrm{x}}$ where $\mathrm{B}$ is the buildup factor. Buildup factor is always equals to or greater than unity.

There are two types of buildup factors - energy absorption buildup factor and exposure buildup factor. In energy absorption buildup factor, the quantity of concern is absorbed or deposited energy in the material and detector response function [DRF] is that of the absorption in the material whereas in exposure buildup factor, the quantity of concern is the exposure of energy and detector response function [DRF] is that of absorption in air.

There are various methods available to compute buildup factors such as G.P. fitting method given by Harima et al. [1], invariant embedding method given by Shimizu and Hirayama [2] and Shimizu et al [3]. American National Standards ANSI/ANS 6.4.3 [4] (American National Standard, 1991) used G.P. fitting method and provided buildup factor data for 23 elements, one compound and two mixtures viz. water, air with suitable interval up to the penetration depth of 40 mean free paths. M.J. Berger and J. Hubbell [5] provided for the first time the database of mass attenuation coefficient as well as cross-sections for about 100 elements in the form of software package named as XCom, which is also capable of generating mass attenuation coefficients for compounds and mixtures. Y. Harima [6] has given an historical review and current status of buildup factor calculations and applications for the materials water, concrete and elements $\mathrm{Fe}, \mathrm{Pb}, \mathrm{Be}, \mathrm{B}, \mathrm{C}, \mathrm{N}, \mathrm{O}, \mathrm{F}, \mathrm{Na}, \mathrm{Mg}, \mathrm{Al}, \mathrm{Si}, \mathrm{P}, \mathrm{S}$, $\mathrm{Ar}, \mathrm{K}, \mathrm{Ca}, \mathrm{Cu}, \mathrm{Mo}, \mathrm{Sn}, \mathrm{La}, \mathrm{Gd}, \mathrm{W}, \mathrm{U}$ in the energy range 0.01 to $0.3 \mathrm{MeV}$ and 0.5 to $10 \mathrm{MeV}$ with penetration depth up to $40 \mathrm{mfp}$, using various codes ADJMOM, PALLAS and ASFIT. H. Hirayama \& K. Shin [7] had used EGS4 Monte Carlo code to study multilayer gamma ray exposure buildup factors up to $40 \mathrm{mfp}$ for water, iron and lead at energies $0.1,0.3,0.6,1.0,3.0,6.0$ and $10 \mathrm{MeV}$.G. S. Sidhu et al. [8] had computed energy

\footnotetext{
a lovedeepgehal@gmail.com
} 
absorption buildup factor for some biological samples viz. Cholesterol, chlorophyll, hemoglobin, muscle, tissue, cell and bone in the energy range of 0.015 to $15.0 \mathrm{MeV}$ with penetration depth up to $40 \mathrm{mfp}$, using G.P. fitting method. Shimizu et al.[9] compared the buildup factor values obtained by three different approaches (G.P. fitting, invariant embedding and Monte Carlo method) and only small discrepancies were observed for low-Z elements up to 100 mean free path. K. Trots et al. [10] proposed vector regression model for the estimation of gamma ray buildup factors for multi-layer shields for $\mathrm{Al}$, $\mathrm{Fe}, \mathrm{Pb}$, water and concrete in the energy range of 5 to 10 $\mathrm{MeV}$ with penetration depth more than $10 \mathrm{mfp}$. P.S. Singh et al. [11] measured variation of energy absorption build up factors with incident photon energy and penetration depth for some commonly used solvents. $\mathrm{T}$. singh et al [12] worked on Chemical composition dependence of exposure buildup factors for some polymers

After going through the above literature, it has been observed that with the ever increasing use of gamma ray photons in medicine and bio-physics, there is a dire need of proper investigations concerning gamma rays multiple scattering effects on Titanium compounds.

In the present work, multiple scattering effects of gamma rays in some compounds of titanium has been studied in terms of some photon interaction parameters viz. mass attenuation coefficient, equivalent atomic number and energy absorption buildup factor in the energy range of $15.0 \mathrm{keV}$ to $15.0 \mathrm{MeV}$ and penetration depth upto 40 $\mathrm{mfp}$.

\section{COMPUTATIONAL WORK}

The computational work i.e. calculation of energy absorption buildup factor has been divided into three parts. In the first step, equivalent atomic numbers are calculated in the incident photon energies ranging from $15.0 \mathrm{keV}$ to $15.0 \mathrm{MeV}$. The second step concerns with the calculation of G.P. fitting parameters using values of equivalent atomic numbers of the selected titanium compounds and finally in the third step, these G.P parameters are then used to calculate absorption buildup factor for the selected titanium compounds.

\subsection{Equivalent atomic numbers $\left(Z_{\text {eq }}\right)$}

Equivalent atomic number is a quantity quite similar to general atomic number of elements which can be defined as the number assigned to a compound or mixture by considering the Compton multiple scattering processes. As we know the buildup factor is a direct consequence of multiple scattering, hence equivalent atomic number $\left(Z_{\mathrm{eq}}\right)$ is used to calculate the buildup factors. In order to calculate $\left(Z_{\text {eq }}\right)$, the values of Compton partial attenuation coefficient $\left(\mu_{\text {comp }}\right)$ and the total attenuation coefficients $\left(\mu_{\text {total }}\right)$ were obtained in $\mathrm{cm}^{2} / \mathrm{g}$ fistly in the energy value from 0.015 to $15.0 \mathrm{MeV}$, using WinXCom computer program (Gerward et al., 2001) The values of $Z_{\text {eq }}$ for the titanium compounds are calculated using following formula:

$$
Z_{e q}=\frac{Z_{1}\left(\log R_{2}-\log R\right)+Z_{2}\left(\log R-\log R_{1}\right)}{\left(\log R_{2}-\log R_{1}\right)}
$$

Where $Z_{1}$ and $Z_{2}$ are the atomic numbers of elements corresponding to the ratios of $\mu_{\text {comp }}$ and $\mu_{\text {total }}, R_{1}$ and $R_{2}$ respectively. $\mathrm{R}\left(\mu_{\text {Comp }} / \mu_{\text {total }}\right)$ is the ratio for the selected titanium compounds at a particular energy value, which lies between ratios $R_{1}$ and $R_{2}$ such that $R_{1}<R<R_{2}$.

\subsection{Computations of G.P. fitting parameters}

ANSI (American National Standard) in 1991 had provided the energy absorption G.P. fitting parameters of 23 elements, one compound $\left(\mathrm{H}_{2} \mathrm{O}\right)$ and two mixtures (air and concrete) in the energy range from 0.015 to 15.0 $\mathrm{MeV}$ and up to a penetration depth of $40 \mathrm{mfp}$. The calculated values of $Z_{\text {eq }}$ earlier were used to interpolate G.P. fitting parameters (b, c, a, $X_{k}$ and d) for the energy absorption buildup factor, in the energy range $(0.015$ $15.0 \mathrm{MeV}$ ) and penetration depth (1-40 mfp). Sidhu et al. (2000) used the formula for the purpose of interpolation of the G.P. fitting parameters as follows:

where

$$
\mathrm{P}=\frac{\mathrm{P}_{1}\left(\log Z_{2}-\log Z_{\mathrm{eq}}\right)+\mathrm{P}_{2}\left(\log Z_{\mathrm{eq}}-\log Z_{1}\right)}{\log Z_{2}-\log Z_{1}}
$$

$\mathrm{Z}_{1}$ and $\mathrm{Z}_{2}$ are the atomic numbers of elements between which the equivalent atomic number $Z_{\mathrm{eq}}$ of the selected titanium compounds lies. Here $\mathrm{P}_{1}$ and $\mathrm{P}_{2}$ are the values of G.P. fitting parameters corresponding to the atomic numbers $Z_{1}$ and $Z_{2}$ respectively at a given energy.

\subsection{Computations of buildup factors}

The computed G.P. fitting parameters (b, c, a, Xk and d) were then used to calculate the energy absorption buildup factors for the selected compounds of titanium at some standard incident photon energies in the range of 0.015 $15.0 \mathrm{MeV}$ and upto a penetration depth of $40 \mathrm{mfp}$, with the help of G.P. fitting formula, as given by following equations (Harima et al., 1986)

$$
\begin{aligned}
& \text { for } \mathrm{K} \neq 1 \quad B(E, X)=1+\frac{b-1}{K-1}\left(K^{X}-1\right) \\
& \begin{array}{l}
\text { for } \mathrm{K}=1 \\
\text { where }
\end{array} \\
& K(E, x)=c x^{a}+d \frac{\tanh \left(\frac{x}{X_{k}}-2\right)-\tanh (-2)}{1-\tanh (-2)} \text { for } \mathrm{X} \leq 40 \mathrm{mfp}
\end{aligned}
$$

\section{RESULTS AND DISCUSSION}

Fig. 3.1-3.4 shows the variation of energy absorption buildup factor with penetration depth of the titanium compounds at incident photon energies $(0.05,0.5,1,5$, 10 and $15 \mathrm{MeV}$ ). For all the titanium compounds, energy absorption buildup factor increases with the increase in penetration depth of the building material. 
It is found that energy absorption buildup factor for the selected compounds of titanium in the energy region of 0.015-15.0 MeV up to the penetration depth of 40 mean free path is always greater than one. This is because with the increase in penetration depth, thickness of the interacting material has been increased which results in increasing the scattering events within the selected titanium compounds. Hence, it results in large energy absorption buildup factor values. For the penetration at large penetration depths, almost similar trend has been observed for the energy absorption buildup factor with incident photon energy.

The dependence of energy absorption buildup factor on penetration depth has been discussed as following:-

Figs. $3.1-3.4$ show the variation of energy absorption buildup factor with incident photon energy for all the selected compounds of titanium at 1, 5, 10, 20, 30 and 40 mean free path respectively., the increasing rate of energy absorption buildup factor for titanium compounds was found to be slow for lower and higher incident photon energies and rapid increase was observed in case of intermediate energy region.

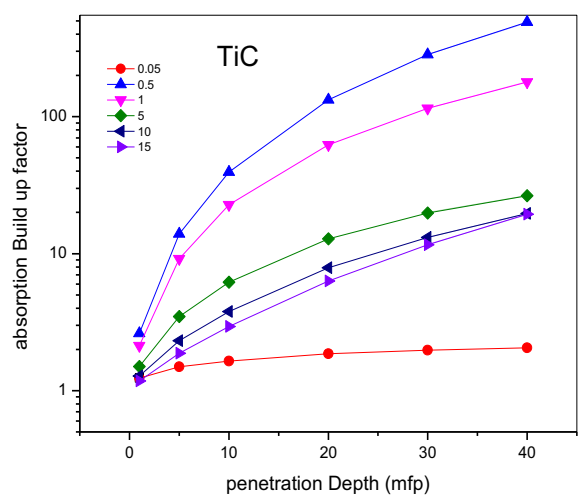

Figure 3.1: Variation of energy absorption buildup factor with penetration depth in case of titanium carbide.

The slower increasing rate in the lower and higher energy regions was due to the dominance of different photon absorption processes in these energy regions (photoelectric effect in the lower energy region and pair production in the higher energy region) which results in the complete absorption of gamma photons in the interacting medium, whereas in the intermediate energy region the dominant process is the Compton scattering, which results only in the energy degradation of photons. Hence, there is finite possibility of the photon to reach the detector even for the large penetration depths of the ceramics and hence maximum violation of Lambert-Beer equation has been observed.

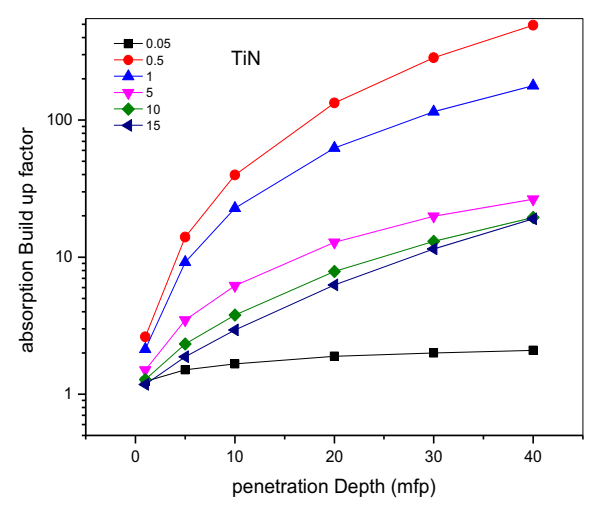

Figure 3.2: Variation of energy absorption buildup factor with penetration depth in case of titanium Nitride.

Further, the increasing rate of energy absorption buildup factor with the penetration depth is more rapid up to the certain incident photon energy $(0.1 \mathrm{MeV})$, where the Compton scattering process is most dominant process and after this increasing rate of energy absorption buildup factor becomes slower for higher energies.

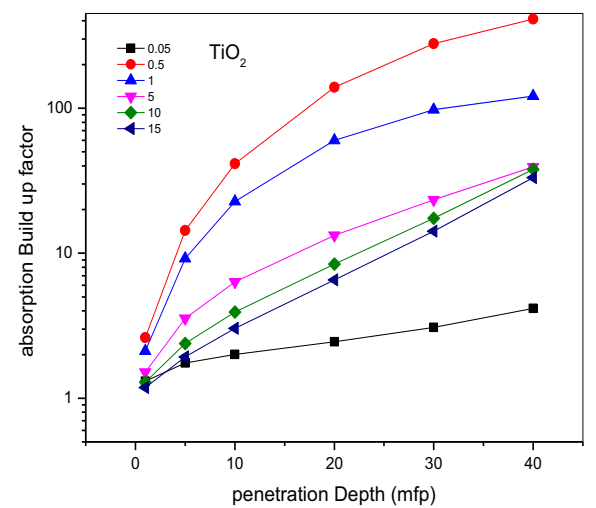

Figure 3.3: Variation of energy absorption buildup factor with penetration depth in case of titanium dioxide.

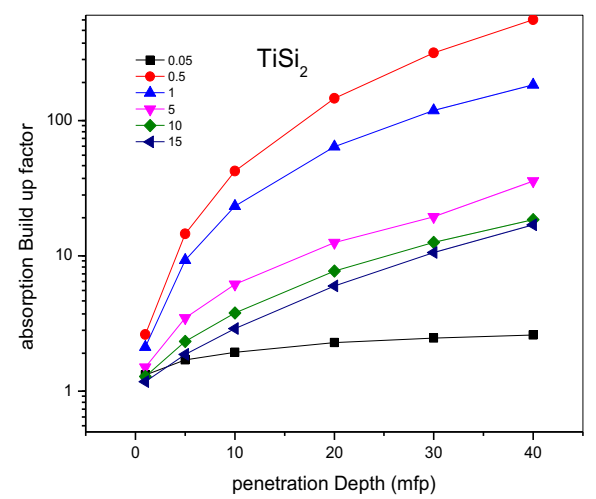

Figure 3.4: Variation of energy absorption buildup factor with penetration depth in case of titanium silicate. 


\section{ACKNOWLEDGMENTS}

Authors are thankful to staff of department of physics, Sant Longowal Institute of Engineering and Technology, Longowal, Sangrur, Punjab, India for their cooperation for doing this work.

\section{References}

1. Harima Y., Tanaka S., Sakamoto Y. \& Hirayama H. 1990. Development of New Gamma-Ray Buildup Factor and Application to Shielding Calculations, Nucl. Sc. \& Tech., 74-84.

2. Shimizu A. \& Hirayama H. 2003, Calculation of gamma-ray buildup factors up to depths of $100 \mathrm{mfp}$ by the method of invariant embedding. Improved treatment of bremsstrahlung. Nucl. Sci. \& Eng. 40, 192-200.

3. Shimizu A. 2002, Calculations of gamma-ray buildup factors up to depth of $100 \mathrm{mfp}$ by the method of invariant embedding. Nucl. Sc. \& Tech. 39, 477-486.

4. American National Standard, ANSI/ANS - 6.4.3. (1991).

5. Berger, M.J., Hubbell, J.H., 1987. N B S I R87-3597: Photon Cross Sections on A Personal Computer.National Institute of Standards and Technology, Gaithersburg, MD.
6. Harima Y. ,1993 An historical review and current status of buildup factor calculations and applications. Radiat. Phy. Chem. 41, 631-672

7. Hirayama H. \& Shin K., 1998 Application of the EGS4 Monte Carlo code to a study of multilayer gamma-ray exposure buildup factors of up to $40 \mathrm{mfp}$. J. Nucl. Sci. Technol. 35, 816-829

8. Sidhu G.S., Singh P.S. \& Mudahar G.S., 1999 Energy absorption buildup factor studies in biological samples. Radiat. Protect. Dosim. 86, 207-216

9. Shimizu A., Onda T. and Sakamato Y., 2004, Calculation of Gamma-Ray Buildup Factors up to Depths of $100 \mathrm{mfp}$ by the Method of Invariant Embedding, (III), . Nucl. Sc. \& Tech, 41, 413-424

10. Trots K. , Smuc T. \& Pevec D. 2007, Support vector regression model for the estimation of gamma ray build up factors for multi-layer shields. Annal. Nucl. Energy. 34, 939-952.

11. Singh P.S., Singh T. and Kaur P. ,2008, Variation of energy absorption build up factors with incident photon energy and penetration depth for some commonly used solvents. Annal. Nucl. Energy, 35, 1093-1097

12. Singh T., Kumar N., Singh P.S. , 2008, Chemical composition dependence of exposure buildup factors for some polymers, . Annal. Nucl. Energy, 36,114120 